OPEN ACCESS

Edited by:

Anja Joachim

Veterinärmedizinische Universität

Wien, Austria

Reviewed by:

Luigi Venco,

Independent Researcher, Pavia, Italy

Giulio Grandi,

Swedish University of Agricultural

Sciences, Sweden

${ }^{*}$ Correspondence:

Paolo E. Crisi

pecrisi@unite.it

Specialty section: This article was submitted to

Parasitology,

a section of the journa

Frontiers in Veterinary Science

Received: 13 December 2017

Accepted: 23 May 2018

Published: 20 June 2018

Citation:

Crisi PE, Di Cesare A and Boari A (2018) Feline Troglostrongylosis:

Current Epizootiology, Clinical Features, and Therapeutic Options.

Front. Vet. Sci. 5:126.

doi: 10.3389/fvets.2018.00126

\section{Feline Troglostrongylosis: Current Epizootiology, Clinical Features, and Therapeutic Options}

\author{
Paolo E. Crisi ${ }^{*}$, Angela Di Cesare and Andrea Boari \\ Faculty of Veterinary Medicine, Veterinary Teaching Hospital, University of Teramo, Teramo, Italy
}

Parasitic bronchopneumonia plays an important role in feline respiratory medicine, thus it is receiving growing attention by researchers and practitioners. In recent years, Troglostrongylus brevior, a lungworm usually infecting wild felids, has been recognized as an agent of the lower respiratory tract in domestic cats. In particular, as a likely consequence of a spill-over from wild reservoirs (e.g., the European wildcat), T. brevior infection is increasingly reported in cats from Mediterranean and Balkan countries. This parasitic nematode has an indirect life cycle, and its biology overlaps that of the better known "cat lungworm" Aelurostrongylus abstrusus. In fact, cases of co-infections caused by both lungworms are not infrequent in domestic cats. Knowledge on clinical features of troglostrongylosis is still incomplete. Available data indicates that clinical signs and radiographic evidence are severe especially in kittens and young cats, are non-specific and often overlap with those of other feline respiratory diseases, such as feline bronchial disease/asthma, or infectious pneumonia. These characteristics make a definitive diagnosis of troglostrongylosis challenging, this disease requires a timely ancillary therapy and an appropriate anthelminthic treatment. As feline troglostrongylosis is an emerging parasitic disease of domestic cats, it should be included in differential diagnosis for lower respiratory tract disease in cats from regions where this parasite is present but also where it is unexpected. This article reviews current knowledge on the pathogenic role of $T$. brevior in domestic cats and resulting respiratory illness, with a special focus on clinical aspects, diagnosis, and management of the disease.

Keywords: troglostrongylosis, Troglostrongylus brevior, lungworms, kitten, wildcat

\section{INTRODUCTION}

Several parasitic nematodes cause infection of the respiratory tract in domestic cats, Aelurostrongylus abstrusus (Strongylida, Angiostrongylidae) being the most common and significant worldwide (1).

Metastrongyloid lungworms ranked within the genus Troglostrongylus, encompassing Troglostrongylus brevior, Troglostrongylus subcrenatus, Troglostrongylus troglostrongylus, and Troglostrongylus wilsoni species, have been considered affiliated only to wild felids for quite a long time (2-6). However, in recent years, the interest of the scientific community on $T$. brevior has grown due to spill-over from wild reservoirs, and recognized ability to cause infection and lower respiratory tract disease in domestic cats (7). 
Troglostrongylus brevior was first described in the last century in two species of wild felids from Palestine (2). It was then found in central Italy in one European wildcat (Felis silvestris silvestris) and in a felid that the author did not clarify whether it was a feral cat or a wildcat (8). No other records of T. brevior were published in peer-reviewed literature until 2010, when this lungworm was described in two domestic cats living in the Ibiza Island (Spain) (9). Since then, an increasing number of reports of T. brevior infections in domestic cats have appeared in literature.

\section{LIFE CYCLE AND EPIZOOTIOLOGY}

Adult worms of $T$. brevior are sexually dimorphic (i.e., male: $6.6-7.2 \mathrm{~mm}$ in length and $0.2-0.23 \mathrm{~mm}$ in width; female $9.6-16.8 \mathrm{~mm}$ in length and $0.26-0.40 \mathrm{~mm}$ in width) and inhabit the bronchi and bronchioles of infected hosts. Females lay eggs hatching first-stage larvae (L1) within the respiratory tract of definitive hosts and a prepatent period of 28 days was observed in an experimentally infected kitten (2).

Troglostrongylus brevior, as other metastrongyloid parasites, needs a mollusc intermediate host to further develop into the infective third-stage larva (L3), and experimental studies show that several slugs and snails, such as Chondrula septemdentata, Helicella barbesiana, Helicella ustalis, Limax flavus, Monaca syriaca, Retinella nitellina, Theba pisana, and Helix aspersa may act as intermediate hosts $(2,10)$.

Paratenic hosts, such as rodents, birds, reptiles, and amphibians, play a pivotal role in the biology and epizootiology of the cat lungworm A. abstrusus (11), however their real significance in the biology of $T$. brevior is not yet wellunderstood. A study showed that a mouse was able to encyst L3 in experimental conditions, but further evidence of development of $T$. brevior in paratenic hosts is still lacking (2). As cats usually do not prey terrestrial molluscs because of their emetic effect, a crucial role of paratenic host in the transmission of T. brevior is more than plausible. Furthermore, a possible vertical transmission of the parasite has been suggested as an alternative route of transmission $(12,13)$.

For a long time, nematodes ranked within the Troglostrongylus genus have been regarded as a parasite of wild felids, such as the leopard cat (Prionailurus bengalensis) (14), bobcat (Lynx rufus) (3, 6), Canada lynx (Felis canadensis) (5), Eurasian lynx (Lynx lynx) (15), leopard (Panthera pardus) (16), tiger (Panthera tigris) (17), jungle cat (Felis chaus) (2), European, and Ethiopian wildcats (Felis sivestris silvestris and Felis ocreata) (2, 18, 19). In domestic cats, after the first report (9), the infection, was reported more and more in cats from Sicily, Sardinia, Crete, Skopelos, Mikonos, and Cyprus Islands (12, 20-24), peninsular and northern Italy $(12,13,25-33)$, continental Greece $(22,33)$, Spain and Bulgaria (34). Recent surveys have shown a prevalence of $T$. brevior second only to the "cat lungworm" A. abstrusus (34), with a prevalence of $14 \%$ in cats from Central Italy (13), 5 and 5.6\% in Cyprus and Greece, respectively (23), and $1.2 \%$ in the Sardinia Island (24). These findings indicate that troglostrongylosis is an up-and-coming disease of the domestic cat across Southern European countries.
The origin of this spread among the population of domestic cats is likely related to a spill-over effect, that occurs when a reservoir population, affected by high pathogen prevalence, interacts with a susceptible one (35). Troglostrongylus brevior has been isolated from domestic cats living in regions where populations of wild cats live in sympatry and are infected by this lungworm with high percentages (36). Furthermore, domestic and wildcats share the same haplotypes in some regions (32), further proving the host switching of $T$. brevior from wild to domestic cats. However, feline troglostrongylosis has been reported also in regions where the natural host responsible for the spread of the infection (i.e., F. s. silvestris) is not documented $(9,33)$. It is possible that movements of paratenic hosts may have introduced infective stages of T. brevior in these areas as well as that cats traveling with their owners from endemic areas to previously free regions could have imported this lungworm (33). Finally, it has been suggested that, in given regions, such as small islands, this parasite has adapted to domestic cats in pre-historical times, before the present coastal arrangement appeared, when the wildcat or a common ancestor was likely present (33).

\section{HISTORY AND CLINICAL SIGNS}

Troglostrongylus brevior infection occurs mostly in cats younger than 1 year $(23,34,36)$, often causing severe and life-threatening lower respiratory tract disease $(12,20,27,31,37)$. Nonetheless, subclinically infected animals and cases of mild clinical signs have been reported, both in young and adult cats $(12,27,31)$. The infection should be suspected in young cats with a history of outdoor life $(9,12,13,20-34)$.

The most common signs in cats with troglostrongylosis are cough, dyspnea and tachypnea $(12,20,22,30,31,37)$. However, clinical signs of lower respiratory tract disease are a common presenting complaint in cats of all ages, and such manifestations frequently overlap with other respiratory disorders (31). Furthermore, other not disease-specific signs, such as anorexia/hyporexia, hyperthermia/hypothermia, dehydration, poor body conditions, and lethargy are reported in feline troglostrongylosis $(22,31)$. Ocular and/or nasal discharge and sneezing are typical signs of feline upper respiratory tract disease (URTD), and these clinical signs have been reported in lungworm and T. brevior infections as well $(20,31)$, even in the absence of the most common pathogens involved in URTD (31). Despite this evidence, the mechanism leading to upper respiratory signs in cats affected by lungworms is still unclear, even though a dislocation of bronchial secretions in the nasal cavity has been proposed (31). However, the concurring presence of infectious agents of URTD and lungworms, especially in young and multi-housed cats, is also likely.

Lung auscultation can reveal increased vesicular breath sounds, usually bilaterally, and wheezing (31). The presence of a right systolic heart murmur has been reported in a 4month old kitten, with a pulmonary hypertension associated with $T$. brevior infection, suggesting an important role of the examination of the cardiocirculatory system, especially 
in those cats with severe respiratory signs, in order to investigate the onset of any possible cardiac complications (30).

\section{DIAGNOSIS}

\section{Hematobiochemical Analysis}

In general, laboratory work in cats with respiratory disease can help to narrow differential diagnosis, to address further tests, to assess co-morbidity, and to give a prognosis. Unfortunately, to date, very few data are available on $\mathrm{CBC}$, serum biochemistry, serum protein electrophoresis, and urinalysis in feline troglostrongylosis and they derive from case reports and case series only $(20,31)$.

The CBC was found within normal limits in the majority of cats affected by $T$. brevior and serum biochemistry examination of these animals was unremarkable $(20,31)$. Peripheral eosinophilia is occasionally observed in cats with parasitic lung disease, but in a recent study, this finding was not observed in 10 cats affected by $T$. brevior, in both single and mixed lungworm infections (31). Leukocytosis is a common finding in feline bronchial disease and it was observed in two 3-month old female kittens with a monospecific infection caused by $T$. brevior $(20,31)$. In one of these cats mild anemia, neutrophilia, and monocytosis, likely as a result of the chronic inflammation, were also observed (31).

\section{Imaging}

Thoracic radiography should be the first assessment in patients with a suspect of lower airway and pulmonary parenchyma disorders. The most common abnormalities detected on chest radiographs in naturally $(31,38,39)$ or experimentally (40) infected cats with lungworm infection are bronchial, nodular, and unstructured interstitial pattern with a multifocal distribution, evolving into a generalized alveolar pattern in severe cases. However, these data relate to A. abstrusus or mixed lungworm infections, and information on imaging features of troglostrogylosis is scant.

Radiographic signs of troglostrongylosis range from mild to severe, and, non-specific patterns, as interstitial, bronchial, and alveolar, either alone or in association, have been reported (Figure 1). Other abnormalities, such as nodular lesions or vascular patterns, were observed in cats with poly-specific infections sustained by $A$. abstrusus and T. brevior $(30,31)$. Interestingly, as already observed in feline bronchopneumonia, including aelurostrongylosis $(38,41,42)$, in two kittens with troglostrongylosis a disagreement, between clinical and radiographic signs, was reported. In particular, interstitial changes were observed in an asymptomatic kitten, while a bronchointerstitial involvement was highlighted in a kitten showing only signs of upper respiratory tract disease (i.e., oculonasal discharge and sneezing), confirming that radiographic changes may be evident before the onset of clinical signs in feline lungworm disease $(31,38)$.

\section{Copromicroscopic Examination and Biomolecular Assays}

For the reasons above a clinical diagnosis is virtually impossible, and clinical, laboratory, and radiographic signs allow only a suspicion of a lower respiratory tract disorder. Thus, a definitive diagnosis of troglostrongylosis can be achieved with observation of parasites or their DNA in biological samples (1).

To date there is no evidence of tracheal wash or BAL cytology as tools for diagnosing troglostrongylosis, but the low sensitivity observed for aelurostrongylosis does not justify the use of this invasive procedure, especially in severe cases (43).

Copromicroscopic examination is a simple, cheap and reliable in-house test, and should always be considered as a first step in the diagnostic work-up for feline lower airway diseases, especially in at-risk subjects (31). As for other metastrongylid parasites, the Baermann test is the gold standard to diagnose feline troglostrongylosis (1). However, this technique has some limitations; first of all, it may be necessary to leave the specimen set up overnight and repeated examinations are necessary to enhance its sensitivity, thus, in some cases, it may take several days. Furthermore, a discrimination between L1s of A. abstrusus, as well as other metastrongyilid larvae (e.g., Oslerus rostratus), and L1s of T. brevior is difficult and requires a careful examination and specific skills (1) (Table 1).

Biomolecular assays make it possible to overcome these limitations and diagnostic methods have been validated for the genetic identification of T. brevior (1). A species-specific nested PCR based on differential markers within the ribosomal (rdNA) internal transcribed spacer 2 region (ITS2) proved effective in identifying $T$. brevior in different biological samples from infected cats (e.g., faces and pharyngeal swabs) (27, 46); furthermore, a duplex PCR assay on markers within ITS2 was developed to discriminate A. abstrusus and T. brevior L1s in a single cat with a mixed infection (26). Finally, a triplex seminested PCR specific for the ITS2 region has been validated for the simultaneous discrimination of A. abstrusus, T. brevior, and Angiostrongylus chabaudi (47).

\section{THERAPY}

\section{Supportive Care}

As mentioned above, dyspnea is a common presentation of feline troglostrongylosis, and in kittens with severe respiratory signs death can occur despite a prompt administration of anthelmintic compounds $(20,27,31,46)$. Thus, in an effort to improve outcomes, supportive care measures are of key importance.

Because stress and handling can exacerbate respiratory signs, care must be taken to not overly stress the cat, and initial treatment may include cage rest and supplemental oxygen. Since these patients, especially kittens, have the ability to decompensate quickly and stress increases the respiratory rate and demand for oxygen, anxiolysis is recommended and, for instance, butorphanol may be given for both anxiolytic and antitussive effects. Furthermore, the administration of shortacting corticosteroids, such dexamethasone sodium phosphate can help to reduce bronchial inflammation (48-50). A fluid 


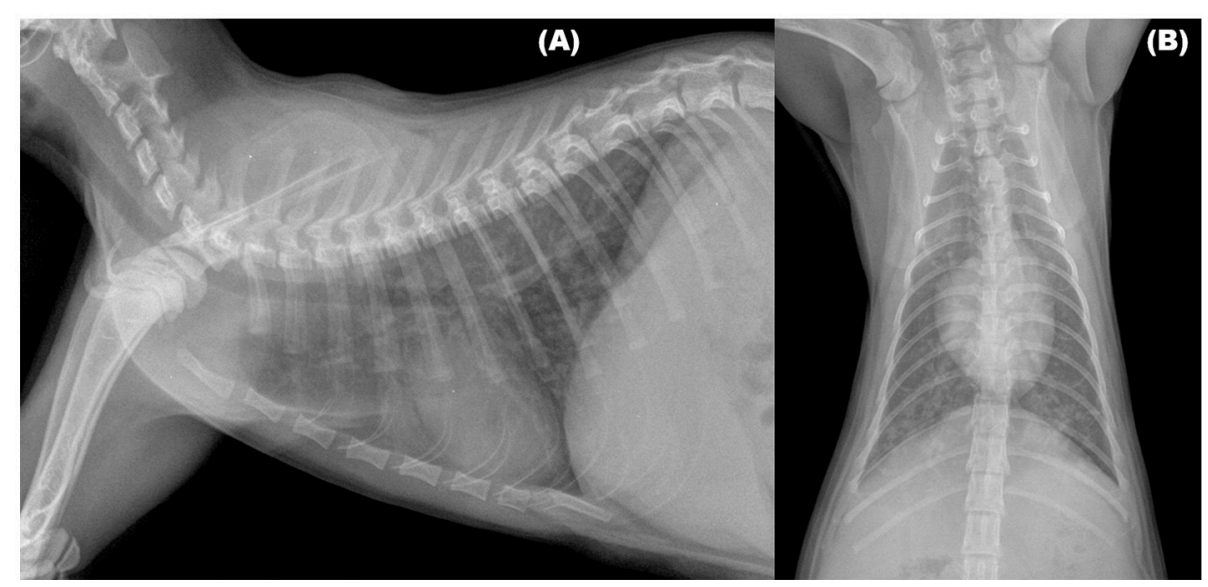

FIGURE 1 | Right lateral (A) and Ventrodorsal (B) views of the thorax of a 3-month old kitten infected by Troglostrongylus brevior. An unstructured interstitial pattern with severe bronchial thickening were visible through the lung lobes.

TABLE 1 | Key features for identification of the first larval stage of Metastrongyloidea in faces of cats.

\begin{tabular}{|c|c|c|c|}
\hline Nematode & Morphological features & Length $(\mu \mathrm{m})$ & References \\
\hline $\begin{array}{l}\text { Aelurostrongylus } \\
\text { abstrusus }\end{array}$ & $\begin{array}{l}\text { Head: rounded, terminal oral opening } \\
\text { Tail: kinked (S-shaped), knob-like or small finger-like } \\
\text { projections at tip of cuticular spines }\end{array}$ & $360-415^{\star}$ & (1) \\
\hline $\begin{array}{l}\text { Troglostrongylus } \\
\text { brevior }\end{array}$ & $\begin{array}{l}\text { Head: pointed, subterminal (dorsal) oral opening } \\
\text { Tail: gradually tapered to the extremity, deep dorsal } \\
\text { incisure and shallower ventral incisure (slight } \\
\text { individual variation) }\end{array}$ & $300-357^{\star}$ & (1) \\
\hline $\begin{array}{l}\text { Troglostrongylus } \\
\text { subcrenatus }\end{array}$ & $\begin{array}{l}\text { Head: pointed, subterminal (dorsal) oral opening } \\
\text { Tail: gradually tapered to the extremity, deep dorsal } \\
\text { incisure and shallower ventral incisure }\end{array}$ & 269-300 & (20) \\
\hline $\begin{array}{l}\text { Oslerus } \\
\text { rostratus }\end{array}$ & $\begin{array}{l}\text { Head: oral opening central to the head and } \\
\text { surrounded by a cuticular ring with dorsal and } \\
\text { ventral prominences } \\
\text { Tail: deep incisure in the ventral side with a cuticular } \\
\text { spine (slight individual variation) }\end{array}$ & $216-412$ & $(34,44)$ \\
\hline $\begin{array}{l}\text { Angiostrongylus } \\
\text { chabaudi** }^{\star 2}\end{array}$ & $\begin{array}{l}\text { Head: rounded, terminal oral opening } \\
\text { Tail: kinked (S-shaped), subterminal spine separated } \\
\text { by a notch, small ventral incisure }\end{array}$ & $362-400$ & (45) \\
\hline
\end{tabular}

*A variation in length of first stage larvae has been reported for Aelurostrongylus abstrusus (minimum $210 \mu m$, maximum $495 \mu \mathrm{m}$ ) and Troglostrongylus brevior (minimum $203 \mu$ m, maximum $382 \mu \mathrm{m})$. ${ }^{\star \star} / a r v a e$ collected from a wildcat (Felis silvestris).

therapy should be considered in those subject with poor clinical conditions (48-50). The role of secondary bacterial infections in lungworm disease is still unknown, and the administration of antibiotics in cats affected by lungworms is questionable and should be evaluated on a case-by-case basis.

\section{Etiological Therapy and Prevention}

At present only spot-on eprinomectin is licensed for treating feline troglostrongylosis, although different compounds have shown promising performance in cats naturally infected with T. brevior.

A single dose of spot-on eprinomectin assured parasitological negativization and clinical recovery in cats with monospecific infection by T. brevior and in cats with T. brevior and A. abstrusus poli-specific infection $(28,34)$.

Spot-on moxidectin was proven effective against T. brevior, in both single and mixed lungworm infections. In particular, a single topical application of moxidectin was effective in assuring parasitological negativization in two kittens with troglostrongylosis. In three cats co-infected by A. abstrusus and T. brevior, two or three moxidectin administrations, 2 weeks apart, were necessary in order to achieve the parasitological negativization. In all such cases, a full clinical recovery was observed in 2-6 weeks after the first administration (31).

No data are currently available on the efficacy of emodepside in treating troglostrongylosis, nevertheless one administration of a spot-on emodepside was effective in assuring clinical recovery 
and reducing larval shedding of $T$. brevior in kittens with a mixed infection, either with A. abstrusus or C. aerophila $(27,31)$. In a further kitten, infected by $T$. brevior in association with A. abstrusus, parasitological negativization and disappearance of clinical signs was recorded after two administrations of emodepside, once every 2 weeks (27).

Clinical signs and larval shedding disappeared after a single oral administration of milbemycin oxime ( $2 \mathrm{mg} / \mathrm{kg}$ body weight) in a kitten with troglostrongylosis and in a kitten infected by T. brevior together with $A$. abstrusus (31).

To date, these compounds have proved their efficacy with an intermediate-weak degree of evidence (i.e., EBM level IV) $(50,51)$ and large-scale studies are necessary to ultimately investigate their efficacy and safety in cats with troglostrongylosis. Nevertheless, different therapeutic options may be used and the anthelminthic compound should be selected based on (i) age and weight of the cats, (ii) indole of the cats, and (iii) owner compliance.

The majority of cats affected by troglostrongylosis are young (i.e., $<2-6$ months) and all of the above-mentioned products are licensed for use in kittens also starting from 6 to 8 weeks of age and a body weight of $0.5-1 \mathrm{~kg}$ (52-55). In general, oral products can be problematical to administer especially in indocile and feral cats while easy-to-apply topical parasiticides are a suitable choice for treating cat lungworms, because of ease of administration, especially when multiple dosing is required $(56,57)$.

Cats totally or partially living outdoors have a high risk to be infected by $T$. brevior, and virtually avoiding external access and subsequently reducing chances of predation is at present the only preventive measure (50). However, this measure is impracticable especially in multi-housed and stray cats. No data are available on chemoprevention of troglostrongylosis, though the potential efficacy of different formulations in the prevention of closely-related metastrongyloid infections was observed. For instance, the formulation containing eprinomectin was able to prevent the infection in experimentally infected cats (58), and spot-on moxidectin is efficacious in preventing the infection by Angiostrongylus vasorum in dogs $(59,60)$. Moxidectin levels are detectable for weeks after treatment (61), and regular administrations of topical moxidectin can induce elevated and sustained steady-state plasma concentrations (52). For instance, in cats, three to five treatments allow to reach a steady-state concentration effective against hookwoorms and Dirofilaria immitis infections $(62,63)$.

\section{REFERENCES}

1. Traversa D, Di Cesare A. Diagnosis and management of lungworm infections in cats: cornerstones, dilemmas and new avenues. J Feline Med Surg. (2016) 18:7-20. doi: 10.1177/1098612X15623113

2. Gerichter CB. Studies on nematodes parasitic in the lungs of felidae in Palestine. Parasitology (1949) 39:251-62. doi: 10.1017/S00311820000 83827

3. Sarmiento L, Stough BD. Troglostrongylus wilsoni (Stough, 1953) n.comb. (Nematoda: Metastrongylidae) from the lungs of the bobcat, Lynx rufus rufus. J Parasitol. (1956) 42:45-8. doi: 10.2307/3274620

\section{PROGNOSIS AND OUTCOME}

A correct diagnosis and an effective anthelminthic therapy often assure a full remission of signs and parasitological negativization within 2-6 weeks (31). Cats receiving outpatient care should be evaluated for clinical condition every week and larval shedding monitored every 2 weeks until clinical and parasitological recovery (31).

Cats, especially young ones, that generally show severe clinical presentation, require hospitalization. These animals can decompensate quickly and may die after an acute respiratory distress, even with intensive therapy and anthelmintic treatment $(20,30,31)$. Thus, in these animals the prognosis is guarded to poor, and the owners should be informed about long-term complications, even rare ones, such as pulmonary hypertension (30).

\section{CONCLUSIONS}

Feline troglostrongylosis is an emergent disease thus, also considering its clinical severity, feline practitioners should be aware of T. brevior in regions where this parasite is present as well as where it is unexpected.

Feline troglostrongylosis should be considered in differential diagnosis for lower respiratory airway disease in cats with outdoor access or in free-roaming animals and copromicroscopic examination should always be considered as a first step in the diagnostic work-up for any cat with respiratory signs. Furthermore, many cats with lower airway diseases may have a subclinical infection and T. brevior, as also other lungworms, can cause radiographic changes even in absence of overt clinical signs (31), indicating that lung damage can be present regardless of the clinical presentation.

\section{AUTHOR CONTRIBUTIONS}

All authors listed have made a substantial, direct and intellectual contribution to the work, and approved it for publication.

\section{ACKNOWLEDGMENTS}

The authors would like to thanks Rosie Trachtman for the valuable support.
4. Watson TG, Nettles VF, Davidson WR. Endoparasites and selected infectious agents in bobcats (Felis rufus) from West Virginia and Georgia. J Wildl Dis. (1981) 17:547-54. doi: 10.7589/0090-3558-17.4.547

5. Smith JD, Addison EM, Joachim DG, Smith LM, Quinn NWS. Helminth parasites of Canada lynx (Felis canadensis) from northern Ontario. Can J Zool. (1986) 64:358-64. doi: 10.1139/z86-057

6. Reichard MV, Caudell DL, Kocan AA. Survey on helminth lung parasites of bobcats (Lynx rufus) from Alabama, Kansas, New Mexico, Oklahoma, and Virginia. U.S.A. Comp Parasitol. (2004) 71:88-90. doi: 10.1654/4086

7. Traversa D, Di Cesare A. Feline lungworms: what a dilemma. Trends Parasitol. (2013) 29:423-30. doi: 10.1016/j.pt.2013.07.004 
8. Paggi L. Segnalazione, in Italia Centrale, di Troglostrongylus sp. parassita dei polmoni di felidi. Parassitologia (1959) 1:80-1.

9. Jefferies R, Vrhovec MG, Wallner N, Catalan DR. Aelurostrongylus abstrusus and Troglostrongylus sp. (Nematoda: Metastrongyloidea) infections in cats inhabiting Ibiza, Spain. Vet Parasitol. (2010) 173:344-48. doi: 10.1016/j.vetpar.2010.06.032

10. Giannelli A, Ramos RA, Annoscia G, Di Cesare A, Colella V, Brianti E, et al. Development of the feline lungworms Aelurostrongylus abstrusus and Troglostrongylus brevior in Helix aspersa snails. Parasitology (2014) 141:56369. doi: 10.1017/S003118201300187X

11. Anderson RC. Nematode Parasites of Vertebrates. Their Development and Transmission. Wallingford: CAB international (2000). p. 605-14.

12. Brianti E, Gaglio G, Napoli E, Falsone L, Giannetto S, Latrofa MS, et al. Evidence for direct transmission of the cat lungworm Troglostrongylus brevior (Strongylida: Crenosomatidae). Parasitology (2013) 140:821-24. doi: $10.1017 /$ S0031182013000188

13. Di Cesare A, Veronesi F, Grillotti E, Manzocchi S, Perrucci S, Beraldo P, et al. Respiratory nematodes in cat populations of Italy. Parasitol Res. (2015) 114:4463-9. doi: 10.1007/s00436-015-4687-5

14. Vevers GM. On the parasitic nematoda collected from mammalian hosts which died in the gardens of the zoological society of london during the years 1919-1921; with a description of three new genera and three new species. Proc Zool Soc Lond. (1922) 92:901-19. doi: 10.1111/j.1469-7998.1922.tb07088.x

15. Alić A, Traversa D, Duscher GG, Kadrić M, Di Cesare A, Hodžić A. Troglostrongylus brevior in an Eurasian lynx (Lynx lynx) from Bosnia and Herzegovina. Parasit Vectors (2015) 8:653. doi: 10.1186/s13071-015-1272-9

16. Cameron TWM. On some lungworms of the malay tiger. J Helmint. (1931) 9:147-52. doi: 10.1017/S0022149X00030388

17. Railliet A, Henry A. Un Haemostrongylus des bronches du Leopard. Bull Soc Pathol Exot Filiales (1913) 4:451-53.

18. Falsone L, Brianti E, Gaglio G, Napoli E, Anile S, Malias, et al. The European wildcats (Felis silvestris silvestris) as reservoir hosts of Troglostrongylus brevior (Strongylida: Crenosomatidae) lungworms. Vet Parasitol. (2014) 205:193-98. doi: 10.1016/j.vetpar.2014.06.024

19. Veronesi F, Traversa D, Lepri E, Morganti G, Vercillo F, Grelli D, et al. Occurrence of Lungworms in European Wildcats (Felis silvestris silvestris) of Central Italy. J Wildl Dis. (2016) 52:270-78. doi: 10.7589/2015-07-187

20. Brianti E, Gaglio G, Giannetto S, Annoscia G, Latrofa MS, Dantas-Torres F, et al. Troglostrongylus brevior and Troglostrongylus subcrenatus (Strongylida: Crenosomatidae) as agents of broncho-pulmonary infestation in domestic cats. Parasit Vectors (2012) 5:178. doi: 10.1186/1756-3305-5-178

21. Tamponi C, Varcasia A, Brianti E, Pipia AP, Frau V, Pinna Parpaglia ML, et al. New insights on metastrongyloid lungworms infecting cats of Sardinia, Italy. D, Scala A. Vet Parasitol. (2014) 203:222-26. doi: 10.1016/j.vetpar.2014.04.001

22. Diakou A, Di Cesare A, Aeriniotaki T, Traversa D. First report of Troglostrongylus brevior in a kitten in Greece. Parasitol Res. (2014) 113:389598. doi: 10.1007/s00436-014-4122-3

23. Diakou A, Sofroniou D, Di Cesare A, Kokkinos P, Traversa D. Occurrence and zoonotic potential of endoparasites in cats of Cyprus and a new distribution area for Troglostrongylus brevior. Parasitol Res. (2017) 116:342935. doi: 10.1007/s00436-017-5651-3

24. Tamponi C, Varcasia A, Pinna S, Melis E, Melosu V, Zidda A, et al. Endoparasites detected in faecal samples from dogs and cats referred for routine clinical visit in Sardinia, Italy. Vet Parasitol. (2017) 10:13-17. doi: 10.1016/j.vprsr.2017.07.001

25. Di Cesare A, Frangipane di Regalbono A, Tessarin C, Seghetti M, Iorio R, Simonato G, et al. Mixed infection by Aelurostrongylus abstrusus and Troglostrongylus brevior in kittens from the same litter in Italy. Parasitol Res. (2014) 113:613-18. doi: 10.1007/s00436-013-3690-y

26. Annoscia G, Latrofa MS, Campbell BE, Giannelli A, Ramos RA, Dantas-Torres F, et al. Simultaneous detection of the feline lungworms Troglostrongylus brevior and Aelurostrongylus abstrusus by a newly developed duplex-PCR. Vet Parasitol. (2014) 199:172-8. doi: 10.1016/j.vetpar.2013.10.015

27. Di Cesare A, Iorio R, Crisi P, Paoletti B, Di Costanzo R, Dimitri CF, et al. Treatment of Troglostrongylus brevior (Metastrongyloidea, Crenosomatidae) in mixed lungworm infections using spot-on emodepside. J Feline Med Surg. (2015) 17:181-5. doi: 10.1177/1098612X14533552
28. Giannelli A, Brianti E, Varcasia A, Colella V, Tamponi C, Di Paola G, et al. Efficacy of Broadline ${ }^{\circledR}$ spot-on against Aelurostrongylus abstrusus and Troglostrongylus brevior lungworms in naturally infected cats from Italy. Vet Parasitol. (2015) 209:273-7. doi: 10.1016/j.vetpar.2015.0 2.037

29. Traversa D, Lepri E, Veronesi F, Paoletti B, Simonato G, Diaferia M, et al. Metastrongyloid infection by Aelurostrongylus abstrusus, Troglostrongylus brevior and Angiostrongylus chabaudi in a domestic cat. Int J Parasitol. (2015) 45:685-90. doi: 10.1016/j.ijpara.2015.05.005

30. Crisi PE, Traversa D, Di Cesare A, Luciani A, Civitella C, Santori $\mathrm{D}$, et al. Irreversible pulmonary hypertension associated with Troglostrongylus brevior infection in a kitten. Res Vet Sci. (2015) 102:223-7. doi: 10.1016/j.rvsc.2015.08.019

31. Crisi PE, Aste G, Traversa D, Di Cesare A, Febo E, Vignoli M, et al. Single and mixed feline lungworm infections: clinical, radiographic and therapeutic features of 26 cases (2013-2015). J Feline Med Surg. (2017) 19:1017-29. doi: $10.1177 / 1098612$ X16670563

32. Traversa D, Veronesi F, Diakou A, Iorio R, Simonato G, Marcer F, et al. Mitochondrial haplotypes of Aelurostrongylus abstrusus and Troglostrongylus brevior (Nematoda, Metastrongyloidea) from domestic and wild felids. Parasitol Res. (2017) 116:1227-35. doi: 10.1007/s00436-017-5 399-9

33. Diakou A, Di Cesare A, Barros LA, Morelli S, Halos L, Beugnet F, et al. Occurrence of Aelurostrongylus abstrusus and Troglostrongylus brevior in domestic cats in Greece. Parasit Vectors (2015) 8:590. doi: 10.1186/s13071-015-1200-z

34. Giannelli A, Capelli G, Joachim A, Hinney B, Losson B, Kirkova Z, et al. Lungworms and gastrointestinal parasites of domestic cats: a European perspective. Int J Parasitol. (2017) 47:517-28. doi: 10.1016/j.ijpara.2017.02.003

35. Power AG, Mitchell CE. Pathogen spillover in disease epidemics. Am Nat. (2004) 164:S79-89. doi: 10.1086/424610

36. Di Cesare A, Veronesi F, Traversa D. Felid lungworms and heartworms in Italy: more questions than answers? Trends Parasitol. (2015) 31:665-75. doi: 10.1016/j.pt.2015.07.001

37. Giannelli A, Passantino G, Ramos RA, Lo Presti G, Lia RP, Brianti $\mathrm{E}$, et al. Pathological and histological findings associated with the feline lungworm Troglostrongylus brevior. Vet Parasitol. (2014) 204:416-9. doi: 10.1016/j.vetpar.2014.05.020

38. Genchi M, Ferrari N, Fonti P, De Francesco I, Piazza C, Viglietti A. Relation between Aelurostrongylus abstrusus larvae excretion, respiratory and radiographic signs in naturally infected cats. Vet Parasitol. (2014) 206:182-7. doi: 10.1016/j.vetpar.2014.10.030

39. Lacava G, Zini E, Marchesotti F, Domenech O, Romano F, Manzocchi S, et al. Computed tomography, radiology and echocardiography in cats naturally infected with Aelurostrongylus abstrusus. J Feline Med Surg. (2017) 19:446-53. doi: 10.1177/1098612X16636419

40. Dennler M, Bass DA, Gutierrez-Crespo B, Schnyder M, Guscetti F, Di Cesare A, et al. Thoracic computed tomography, angiographic computed tomography, and pathology findings in six cats experimentally infected with Aelurostrongylus abstrusus. Vet Radiol Ultrasound. (2013) 54:459-69. doi: $10.1111 /$ vru. 12044

41. Suter PF, Lord PF. Radiographic differentiation of disseminated pulmonary parenchymal diseases in dogs and cats. Vet Clin North Am. (1974) 4:687-710.

42. Losonsky JM, Smith FG, Lewis RE. Radiographic findings of Aelurostrongylus abstrusus infection in cats. J Am Anim Hosp Assoc. (1978) 14:348-55.

43. Lacorcia L, Gasser RB, Anderson GA, Beveridge I. Comparison of bronchoalveolar lavage fluid examination and other diagnostic techniques with the Baermann technique for detection of naturally occurring Aelurostrongylus abstrusus infection in cats. J Am Vet Med Assoc. (2009) 235:43-9. doi: 10.2460/javma.235.1.43

44. Seneviratna P. Studies on Anafilaroides rostratus Gerichter, 1949 in cats. II. The life cycle. J Helminthol. (1959) 33:109-22.

45. Diakou A, Psalla D, Migli D, Di Cesare A, Youlatos D, Marcer F, Traversa D. First evidence of the European wildcat (Felis silvestris silvestris) as definitive host of Angiostrongylus chabaudi. Parasitol Res. (2016) 115:1235-44. doi: 10.1007/s00436-015-4860-x

46. Traversa D, Romanucci M, Di Cesare A, Malatesta D, Cassini R, Iorio R, et al. Gross and histopathological changes associated with Aelurostrongylus 
abstrusus and Troglostrongylus brevior in a kitten. Vet Parasitol. (2014) 201:158-62. doi: 10.1016/j.vetpar.2014.01.020

47. Di Cesare A, Veronesi F, Frangipane di Regalbono A, Iorio R, Traversa D. Novel molecular assay for simultaneous identification of neglected lungworms and heartworms affecting cats. J Clin Microbiol. (2015) 53:300913. doi: 10.1128/JCM.00901-15

48. Mandell DC. Respiratory distress in cats. In: King LG, editor. Textbook of Respiratory Disease in Dogs and Cats. Philadelphia, PA: WB Saunders (2004). p. 12-7.

49. Rozanski E, Chan DL. Approach to the patient with respiratory distress. Vet Clin Small Anim Pract. (2005) 35:307-17. doi: 10.1016/j.cvsm.2004.12.003

50. Pennisi MG, Hartmann K, Addie DD, Boucraut-Baralon C, Egberink $\mathrm{H}$, Frymus $\mathrm{T}$, et al. Lungworm disease in cats: $\mathrm{ABCD}$ guidelines on prevention and management. J Feline Med Surg. (2015) 17:626-36. doi: $10.1177 / 1098612$ X15588455

51. Lloret A. The process of evidence-based medicine. J Feline Med Surg. (2009) 11:529. doi: 10.1016/j.jfms.2009.05.001

52. European Medicines Agency: Advocate- EMEA/V/C/000076 -IAIN/0038 (2017). Available online at: http://www.ema.europa.eu/docs/en_GB/ document_library/EPAR_-_Product_Information/veterinary/000076/ WC500060917

53. European Medicines Agency: Profender -EMEA/V/C/000097 -IAIN/0041 (2017). Available online at: http://www.ema.europa.eu/docs/en_GB/ document_library/EPAR_-_Product_Information/veterinary/000097/ WC500063851

54. European Medicines Agency: Broadline -EMEA/V/C/002700 -IB/0016/G (2017). Available online at: http://www.ema.europa.eu/docs/en_GB/ document_library/EPAR_-_Product_Information/veterinary/002700/ WC500163991

55. Summary of Product Characteristics of Milbemax Film-Coated Tablets for Small Cats and Kittens (2007). Available online at: http://mri.cts-mrp.eu/download/ FR_V_0135_003_FinalSPC.pdf

56. Traversa D, Di Cesare A, Milillo P, Lohr B, Iorio R, Pampurini F, et al. Efficacy and safety of imidacloprid 10\%/moxidectin 1\% spot-on formulation in the treatment of feline aelurostrongylosis. Parasitol Res. (2009) 105:S55-62. doi: 10.1007/s00436-009-1496-8

57. Traversa D, Milillo P, Di Cesare A, Lohr B, Iorio R, Pampurini F, et al. Efficacy and safety of emodepside $2.1 \%$ /praziquantel $8.6 \%$ spot-on formulation in the treatment of feline aelurostrongylosis. Parasitol Res. (2009) 105:S83-9. doi: 10.1007/s00436-009-1499-5
58. Knaus M, Chester ST, Rosentel J, Kühnert A, Rehbein S. Efficacy of a novel topical combination of fipronil, (S)-methoprene, eprinomectin and praziquantel against larval and adult stages of the cat lungworm, Aelurostrongylus abstrusus. Vet Parasitol. (2014) 202:64-8. doi: 10.1016/j.vetpar.2014.02.042

59. Schnyder M, Fahrion A, Ossent P, Kohler L, Webster P, Heine J, et al. Larvicidal effect of imidacloprid/moxidectin spot-on solution in dogs experimentally inoculated with Angiostrongylus vasorum. Vet Parasitol. (2009) 166:326-32. doi: 10.1016/j.vetpar.2009. 09.004

60. Böhm C, Petry G, Schmidt H, Raue K, Barthel F, Schaper R. Evaluation of the persistent preventive efficacy of $2.5 \%$ moxidectin $/ 10 \%$ imidacloprid spot-on (Advocate ${ }^{\circledR}$, Advantage ${ }^{\circledR}$ Multi) in dogs experimentally infected with Angiostrongylus vasorum. Parasitol Res. (2017) 116:1-10. doi: 10.1007/s00436-017-5487-x

61. Prichard R, Ménez C, Lespine A. Moxidectin and the avermectins: consanguinity but not identity. Int J Parasitol Drugs Drug Resist. (2012) 2:134-53. doi: 10.1016/j.ijpddr.2012.04.001

62. Taweethavonsawat $P$, Chungpivat $S$, Watanapongchati $S$, Traub RJ, Schaper R. Efficacy of a spot on combination containing imidacloprid $10 \%$ and moxidectin $1 \%$ (Advocate ${ }^{\circledR} /$ Advantage ${ }^{\circledR}$ Multi, Bayer Animal Health) against Ancylostoma ceylanicum in cats. Vet Parasitol. (2012) 190:289-93. doi: 10.1016/j.vetpar.2012.05.008

63. Little SE, Hostetler JA, Thomas JE, Bailey KL, Barrett AW, Gruntmeir K, et al. Administration of $10 \%$ imidacloprid- $1 \%$ moxidectin protects cats from subsequent, repeated infection with Dirofilaria immitis. In: Proceedings of the 59th Annual Meeting of the American Association of Veterinary Parasitologists Abstract 49. Denver, CO (2014). p. 26-9.

Conflict of Interest Statement: The authors declare that the research was conducted in the absence of any commercial or financial relationships that could be construed as a potential conflict of interest.

Copyright () 2018 Crisi, Di Cesare and Boari. This is an open-access article distributed under the terms of the Creative Commons Attribution License (CC $B Y)$. The use, distribution or reproduction in other forums is permitted, provided the original author(s) and the copyright owner are credited and that the original publication in this journal is cited, in accordance with accepted academic practice. No use, distribution or reproduction is permitted which does not comply with these terms. 